\title{
Recognition of Sequences of Graphical Patterns
}

\author{
Edmondo Trentin ${ }^{1}$, ShuJia Zhang ${ }^{2}$, and Markus Hagenbuchner ${ }^{2}$ \\ ${ }^{1}$ DII - Università di Siena, V. Roma, 56 Siena, Italy \\ trentin@dii.unisi.it \\ ${ }^{2}$ University of Wollongong, Wollongong, 2522 NSW, Australia \\ $\{$ sz603, markus\}@uow. edu. au
}

\begin{abstract}
Several real-world problems (e.g., in bioinformatics/proteomics, or in recognition of video sequences) can be described as classification tasks over sequences of structured data, i.e. sequences of graphs, in a natural way. This paper presents a novel machine that can learn and carry out decision-making over sequences of graphical data. The machine involves a hidden Markov model whose state-emission probabilities are defined over graphs. This is realized by combining recursive encoding networks and constrained radial basis function networks. A global optimization algorithm which regards to the machine as a unity (instead of a bare superposition of separate modules) is introduced, via gradient-ascent over the maximum-likelihood criterion within a Baum-Welch-like forward-backward procedure. To the best of our knowledge, this is the first machine learning approach capable of processing sequences of graphs without the need of a pre-processing step. Preliminary results are reported.
\end{abstract}

Keywords: Hidden Markov model, relational learning, recursive networks.

\section{Introduction}

This paper introduces a novel hybrid architecture (along with its training algorithm) for learning over sequences of graphs (i.e., sequential structured data). The scenario is an extension of traditional relational learning, where a learning machine is fed with a graph (an algebraic relation), and it is expected to carry out classification (or, regression) over the input structured data [5]. In the present setup, we are faced with a sequence $g_{1}, \ldots, g_{n}$ of individual graphs, and the overall sequence has to be modeled and classified. Examples may help focusing on the nature of this scenario. Several problems in bioinformatics concern the classification of proteins, or of subparts of proteins. For instance, prediction of the secondary structure of certain segments within the protein, or the prediction of the binding state of individual cysteines, starting from the primary structure. These problems are usually faced (e.g., using hidden Markov models) relying on a description of the protein as a sequence (string) of amino-acids, each amino-acid being a symbol drawn from a finite and discrete alphabet, or a real-valued feature vector drawn from a multiple-alignment profile. In so doing, the very nature of the amino-acids is overlooked by the machine, which has no opportunity to take benefit from knowledge of the underlying physical and chemical properties of the amino-acids themselves. As a matter of fact, each amino-acid is a molecule, built up from specific atoms (having specific properties) which are implicitly involved in a binary relation defined by the 
atom-atom chemical bonds. Such molecules may be represented as labeled graphs in a natural manner. Overall, the whole primary structure of the protein can thus be described in terms of a sequence of graphs, each representing the amino-acid that is found at that specific location along the sequence. A machine which is capable of dealing with such a sequence of graphs, and capable of carrying out an automatic segmentation of the protein into relevant sub-sequences (segments), may exploit the knowledge encapsulated in the sub-molecular properties of the polymer.

Another example is found in the area of video processing. Relational learning machines, such as recursive neural nets (RNN) [8], have been widely applied to image classification tasks. In this case, a graphical representation of images is extracted, e.g. in terms of region adjacency graphs (RAG) [3], or multi-resolution trees (MRT) [2]. The idea is that a graphical representation is richer than a traditional, flat feature vector representation. Given that a video consists of a sequence of still images, and hence, it is quite obvious that a video is suitably represented as a sequence of graphs (either RAGs or MRTs). Again, a machine capable of learning and accomplishing classification over sequences of graphs is sought.

More examples can be found in the areas of molecular chemistry, document processing, the World Wide Web, environmental computing, etc. whenever there is a time dependency between structured objects.

This paper introduces a novel machine that fits the framework of sequential graphical pattern recognition. The architecture is defined as follows. The hidden part of a hidden Markov model (HMM) [7] is taken, along with its intrinsic capability of modeling long-term time dependencies, and of performing automatic segmentation of long sequences into sub-sequences. The emission probabilities associated with the states of the HMM are probability density functions (PDF) defined over labeled graphs. Emission PDFs are modeled using a combined artificial neural network (ANN) architecture. An encoding ANN, as in RNNs for graphs [8], is combined with a radial basis function (RBF)-like network that realizes the estimation of the PDF. This probabilistic interpretation is made possible by (i) a description of individual graphs as the random outcomes of a generalized random graph, as was formally defined in [9]; and by (ii) a constrained RBF which actually realizes a PDF model which satisfies probability axioms. We stress the fact that the resulting, hybrid machine is not just the aggregation of separate, cooperating architectures. Rather, it shall be thought of as a whole, since a global, joint optimization algorithm is developed, which trains all the model parameters (RBFs parameters, encoding networks weights, HMM initial and transition probabilities) simultaneously in order to increase a shared, overall criterion function, namely the maximum-likelihood (ML) of the model given a sample of training observation sequences. Training takes place within the popular Forward-backward (or, Baum-Welch) procedure for HMMs [7]. Once training is accomplished, the popular Viterbi algorithm [7] can be applied in order to carry out segmentation and classification of sequences of graphical patterns.

The paper is organized as follows: Section 2 gives the fundamental mathematical details of the algorithm. An application to a learning problem is described in Section 3 , and some concluding remarks are made in Section 4

\footnotetext{
${ }^{1}$ A formal notion of PDF over labeled graphs is given in the next Section.
} 


\section{Architecture and Training Algorithm}

We will use the notion of generalized random graph [9] for a formal definition of the probabilistic quantities over sequences of graphs that underly the given framework. Let $\mathcal{V}$ be a given discrete- or continuous-valued set (vertex universe), and let $\Omega$ be any given sample space. We define a Generalized Random Graph (GRG) over $\mathcal{V}$ and $\Omega$ as a function $G: \Omega \rightarrow\{(V, E) \mid V \subseteq \mathcal{V}, E \subseteq V \times V\}$. Let then $\mathcal{G}=\{(V, E) \mid V \subseteq$ $\mathcal{V}, E \subseteq V \times V\}$. A probability density function (PDF) for GRGs over $\mathcal{V}$ is a function $p: \mathcal{G} \rightarrow \Re$ such that: (1) $p(g) \geq 0, \forall g \in \mathcal{G}$, and (2) $\int_{\mathcal{G}} p(g) d g=1$ (refer to [9] for a discussion on measurability of GRG spaces, i.e. meaning of this integral). Loosely speaking, any function $G()=.\xi(t)$ which maps time $t$ (either discrete or continuous) onto a GRG is then defined to be a stochastic graph process. In turn, a hidden Markov model over graphs (GHMM) is a pair of stochastic processes: a hidden Markov chain 2 and an observable stochastic graph process which is a probabilistic function of the states of the former. More precisely, a GHMM is defined as a traditional HMM [7] except for the notion of emission probability, namely as:

1. A set $S$ of $Q$ states, $S=\left\{S_{1}, \ldots, S_{Q}\right\}$, which are the distinct values that the discrete, hidden stochastic process can take.

2. An initial state probability distribution, i.e. $\pi=\left\{\operatorname{Pr}\left(S_{i} \mid t=0\right), S_{i} \in S\right\}$, where $t$ is a discrete time index.

3. A probability distribution that characterizes the allowed transitions between states, that is $\mathbf{a}_{i j}=\left\{\operatorname{Pr}\left(S_{j}\right.\right.$ at time $t+1 \mid S_{i}$ at time $\left.\left.t\right), S_{i} \in S, S_{j} \in S\right\}$ where the transition probabilities $\mathbf{a}_{i j}$ are assumed to be independent of time $t$. Note that $\left\{\operatorname{Pr}\left(S_{j}\right.\right.$ at time $t+1 \mid S_{i}$ at time $t, S_{k}$ at time $\left.t-1, \ldots\right)=\mathbf{a}_{i j}$ due to the Markov assumption.

4. A set of PDFs over GRGs (referred to as emission probabilities) that describes the statistical properties of the GRGs for each state of the model: $\mathbf{b}_{\mathbf{G}}=\left\{b_{i}(g)=p(g \mid\right.$ $\left.\left.S_{i}\right), S_{i} \in S, g \in \mathcal{G}\right\}$.

Let us assume that a certain sequence $Y=g_{1}, \ldots, g_{T}$ of graphs generated by a (hidden) stochastic graph process has been observed, and that it is the expression (outcome) of a certain sequence $\mathcal{W}=\omega_{1}, \ldots, \omega_{L}$ of states of nature (i.e., classes). Recognition (classification) of the correct class(es) $\mathcal{W}$ relying on the observations $Y$ can be accomplished according to the class(es) posterior probability given $Y$, yielded by Bayes' theorem: $\operatorname{Pr}(\mathcal{W} \mid Y)=p(Y \mid \mathcal{W}) \operatorname{Pr}(\mathcal{W}) / p(Y)$. The quantity $\operatorname{Pr}(\mathcal{W})$ is referred to as the prior probability of $\mathcal{W}$. It can be estimated from relative frequencies of classes as in statistical pattern recognition. We propose GHMMs for modeling the class-conditional density $p(Y \mid \mathcal{W})$. Note that this approach deals with "continuous" recognition tasks, that is a sequence of classes $\omega_{1}, \ldots, \omega_{L}$ is hidden behind the observations $Y$, and no prior segmentation of $Y$ into subsequences $Y_{1}, \ldots, Y_{L}$ corresponding with the individual classes in known in advance.

The proposed machine relies on a connectionist non-parametric model of the emission probabilities of a GHMM, with gradient-ascent global training techniques over the ML criterion. An ANN is introduced for each state of the GHMM. The output

\footnotetext{
${ }^{2}$ This is a traditional, discrete time random process.
} 
unit of a generic ANN provides an estimate of the corresponding emission probability (that is, a PDF over GRGs) given the current graphical observation in the input graph space. Training of the other probabilistic quantities in the underlying Markov chain, i.e. initial and transition probabilities, still relies on likelihood maximization via the forward-backward algorithm [7]. The Viterbi algorithm is then applied to the recognition step [7].

The global criterion function to be maximized during training, namely the likelihood $L$ of a graphical observation sequence given the mode 3 , is defined as $L=\sum_{\iota \in \mathcal{F}} \alpha_{\iota, T}$. The sum is extended to the set $\mathcal{F}$ of all possible final states [1] within the GHMM corresponding to the current training sequence. The GHMM is supposed to involve $Q$ states, and $T$ is the length of the current observation sequence $Y=g_{1}, \ldots, g_{T}$. The forward terms $\alpha_{\iota, t}=\operatorname{Pr}\left(q_{\iota, t}, g_{1}, \ldots, g_{t}\right)$ and the backward terms $\beta_{\iota, t}=\operatorname{Pr}\left(g_{t+1}, \ldots, g_{T} \mid q_{\iota, t}\right)$ for $\iota$-th state at time $t$ can be computed recursively as follows [7]:

$$
\alpha_{\iota, t}=b_{\iota, t} \sum_{j} a_{j \iota} \alpha_{j, t-1}
$$

and

$$
\beta_{\iota, t}=\sum_{j} b_{j, t+1} a_{\iota j} \beta_{j, t+1}
$$

where $a_{\iota j}$ denotes the transition probability from the $\iota$-th state to the $j$-th state, $b_{\iota, t}$ denotes the emission probability associated with the $\iota$-th state over the $t$-th graph $g_{t}$, and the sums are extended to all possible states within the GHMM. The initialization of the forward probabilities is accomplished as in HMMs [7], whereas the backward terms at time $T$ are initialized in a slightly different manner, namely:

$$
\beta_{\iota, T}=\left\{\begin{array}{l}
1 \text { if } \iota \in \mathcal{F} \\
0 \text { otherwise. }
\end{array}\right.
$$

Given a generic parameter $\theta$ of an ANN, hill-climbing gradient-ascent over $L$ prescribes a learning rule of the following well-known kind:

$$
\Delta \theta=\eta \frac{\partial L}{\partial \theta}
$$

where $\eta \in \Re^{+}$, and $\eta$ is commonly known as the learning rate. Let us observe (from [1]) that the following property can be easily shown to hold true by taking the partial derivatives of the left- and right-hand sides of Equation (1) with respect to $b_{\iota, t}$ :

$$
\frac{\partial \alpha_{\iota, t}}{\partial b_{\iota, t}}=\frac{\alpha_{\iota, t}}{b_{\iota, t}} .
$$

In addition, by borrowing the scheme proposed by [1], the following theorem can be proved to hold true: $\frac{\partial L}{\partial \alpha_{\iota, t}}=\beta_{\iota, t}$, for each $\iota=1, \ldots, Q$ and for each $t=1, \ldots, T$.

\footnotetext{
${ }^{3}$ A standard notation is used in the following to refer to quantities involved in HMM training (e.g. [7]). Note that the Greek letter $\iota$ (iota) is used to denote the index for a generic state $q_{\iota}$ of the GHMM; it shall not be confused with the index $i$ that will be introduced later.
} 
Given this theorem and Equation (5), repeatedly applying the chain rule we can expand $\frac{\partial L}{\partial \theta}$ by writing:

$$
\begin{aligned}
\frac{\partial L}{\partial \theta} & =\sum_{q} \sum_{t} \frac{\partial L}{\partial b_{q, t}} \frac{\partial b_{q, t}}{\partial \theta} \\
& =\sum_{q} \sum_{t} \frac{\partial L}{\partial \alpha_{q, t}} \frac{\partial \alpha_{q, t}}{\partial b_{q, t}} \frac{\partial b_{q, t}}{\partial \theta} \\
& =\sum_{q} \sum_{t} \beta_{q, t} \frac{\alpha_{q, t}}{b_{q, t}} \frac{\partial b_{q, t}}{\partial \theta}
\end{aligned}
$$

where the sums are extended over all states $q$ of the GHMM involved in the current training sequence (i.e., all the rows in the current trellis [7]), and to all $t=1, \ldots, T$, respectively. It is seen that all the quantities in the right-hand side of Equation (6) are available upon recursive processing on the standard HMM trellis, except for $\frac{\partial b_{q, t}}{\partial \theta}$. From now on, attention is thus focused on the calculation of $\frac{\partial b_{q, t}}{\partial \theta}$, where $b_{q, t}$ is the output from the corresponding ANN at time $t$.

Now, for each $\iota=1, \ldots, Q$ let us assume the existence of an integer $d$ and of two functions, $\phi_{\iota}: \mathcal{G} \rightarrow \Re^{d}$ and $p_{\iota}: \Re^{d} \rightarrow \Re$, s.t. $b_{\iota, t}$ can be decomposed as:

$$
b_{\iota, t}=p_{\iota}\left(\phi_{\iota}\left(g_{t}\right)\right)
$$

We call $\phi_{\iota}($.$) the encoding for \iota$-th state of the GHMM, while $p_{\iota}($.$) is simply referred$ to as the "emission" associated with that state 4 . Again, we assume parametric forms $\phi_{\iota}\left(g_{t} \mid \boldsymbol{\theta}_{\phi_{\iota}}\right)$ and $p_{\iota}\left(\mathbf{x} \mid \boldsymbol{\theta}_{p_{\iota}}\right)$ for the encoding and for the emission, respectively, and we set $\boldsymbol{\theta}_{\iota}=\left(\boldsymbol{\theta}_{\phi_{\iota}}, \boldsymbol{\theta}_{p_{\iota}}\right)$. Bearing in mind Equation (7), we propose a state-specific, twoblock connectionist/statistical model for $b_{\iota, t}$ as follows. The function $\phi_{\iota}\left(g_{t} \mid \boldsymbol{\theta}_{\phi_{\iota}}\right)$ is realized via an encoding network, suitable to map graphs $g_{t}$ into real vectors $\mathbf{x}_{t}$ for $t=1, \ldots, T$, as described in [8] for supervised training of RNNs over structured domains. The weights of the encoding network are the parameters $\boldsymbol{\theta}_{\phi_{\iota}}$. A radial basis functions (RBF)-like neural net is then used to model the emission $p_{\iota}\left(\mathbf{x}_{t} \mid \boldsymbol{\theta}_{p_{\iota}}\right)$, where $\boldsymbol{\theta}_{p_{\iota}}$ are the parameters of the RBF. Basically, for each state $\iota$ in the GHMM a statespecific RBF is expected to define a mixture of Normal densities over the state-specific encoding $\phi_{\iota}\left(g_{t} \mid \boldsymbol{\theta}_{\phi_{\iota}}\right)$ of $t$-th input graph. From now on, since (i) HMMs assume that the emission probabilities associated with different states are independent of each other [7], (ii) a separate connectionist model is adopted for each one of the states of the GHMM, and (iii) also individual observations (i.e., graphs) within the input sequence are assumed to be independent of each other given the state [7], we simplify the (cumbersome) notation by dropping the state index $\iota$ and the time index $t$, and we focus on the generic quantity $\left.p\left(\phi\left(g \mid \boldsymbol{\theta}_{\phi}\right)\right) \mid \boldsymbol{\theta}_{p}\right)$. Note that, for notational convenience, in the following this quantity may be written in short as $p(g)$.

\footnotetext{
${ }^{4}$ It is seen that there exist (infinite) choices for $\phi($.$) and \hat{p}($.$) that satisfy Equation (7), the most$ trivial being $\phi(g)=p(g \mid \boldsymbol{\theta}), \hat{p}(x)=x$.
} 
In order to ensure that a PDF is obtained this way, a standard RBF cannot be used straightforwardly: specific constraints have to be placed on the nature of the Gaussian kernels, as well as on the hidden-to-output connection weights. The training algorithm shall provide us with a likelihood maximization scheme that undergoes such constraints. Three distinct families of adaptive parameters $\theta$ of the ANNs have to be considered:

(1) Mixing parameters $c_{1}, \ldots, c_{n}$, i.e. the hidden-to-output weight of the RBF network. Constraints have to be placed on these parameters during the ML estimation process, in order to ensure that they are in the range $(0,1)$ and that they sum to one. A simple way to satisfy the requirements is to introduce $n$ hidden parameters $\gamma_{1}, \ldots, \gamma_{n}$, which are unconstrained, and to set

$$
c_{i}=\frac{\varsigma\left(\gamma_{i}\right)}{\sum_{j=1}^{n} \varsigma\left(\gamma_{j}\right)}, i=1, \ldots, n
$$

where $\varsigma(x)=1 /\left(1+e^{-x}\right)$. Each $\gamma_{i}$ is then treated as an unknown parameter $\theta$ to be estimated via ML.

(2) The $d$-dimensional mean vector $\mu_{i}$ and $d \times d$ covariance matrix $\Sigma_{i}$ for each one of the Gaussian kernels $K_{i}(\mathbf{x})=N\left(\mathbf{x} ; \mu_{i}, \Sigma_{i}\right), i=1, \ldots, n$ of the RBF-like network, where $N\left(\mathbf{x} ; \mu_{i}, \Sigma_{i}\right)$ denotes a multivariate Normal PDF having mean vector $\mu_{i}$, covariance matrix $\Sigma_{i}$, and evaluated over the random vector $\mathbf{x}$. A common (yet effective) simplification is to consider diagonal covariance matrices, i.e. independence among the components of the input vector $\mathbf{x}$. This assumption leads to the following three major consequences: (i) modeling properties are not affected, according to [6]; (ii) generalization capabilities of the overall model may turn out to be improved, since the number of free parameters is reduced to a significant extent; (iii) $i$-th multivariate kernel $K_{i}$ may be expressed in the form of a product of $d$ univariate Normal densities as:

$$
K_{i}(\mathbf{x})=\prod_{j=1}^{d} \frac{1}{\sqrt{2 \pi} \sigma_{i j}} \exp \left\{-\frac{1}{2}\left(\frac{x_{j}-\mu_{i j}}{\sigma_{i j}}\right)^{2}\right\}
$$

i.e., the free parameters to be estimated are the means $\mu_{i j}$ and the standard deviations $\sigma_{i j}$, for each kernel $i=1, \ldots, n$ and for each component $j=1, \ldots, d$ of the input space.

(3) The weights $\mathcal{U}$ of the encoding network.

In the following, we will derive explicit formulations for $\frac{\partial p\left(\phi\left(g \mid \boldsymbol{\theta}_{\phi}\right) \mid \boldsymbol{\theta}_{p}\right)}{\partial \theta}$ for each of the three families of free parameters $\theta$ above. These derivatives are then put in place of $\frac{\partial b_{q, t}}{\partial \theta}$ in Equation (6), obtaining the quantity $\frac{\partial L}{\partial \theta}$ and, in turn, the overall learning rule $\Delta \theta=\eta \frac{\partial L}{\partial \theta}$ for the generic parameter $\theta$.

As regards a generic mixing parameter $c_{i}, i=1, \ldots, n$, from Equations (7) and (8), and since $p(g)=\sum_{k=1}^{n} c_{k} K_{k}(\mathbf{x})$, we can obtain Equation 10 


$$
\begin{aligned}
\frac{\partial p\left(\phi\left(g \mid \boldsymbol{\theta}_{\phi}\right) \mid \boldsymbol{\theta}_{p}\right)}{\partial \gamma_{i}} & =\sum_{j=1}^{n} \frac{\partial p(g)}{\partial c_{j}} \frac{\partial c_{j}}{\partial \gamma_{i}} \\
& =\sum_{j=1}^{n} K_{j}(\mathbf{x}) \frac{\partial}{\partial \gamma_{i}}\left(\frac{\varsigma\left(\gamma_{j}\right)}{\sum_{k=1}^{n} \varsigma\left(\gamma_{k}\right)}\right) \\
& =K_{i}(\mathbf{x})\left\{\frac{\varsigma^{\prime}\left(\gamma_{i}\right) \sum_{k} \varsigma\left(\gamma_{k}\right)-\varsigma\left(\gamma_{i}\right) \varsigma^{\prime}\left(\gamma_{i}\right)}{\left[\sum_{k} \varsigma\left(\gamma_{k}\right)\right]^{2}}\right\}+\sum_{j \neq i} K_{j}(\mathbf{x})\left\{\frac{-\varsigma\left(\gamma_{j}\right) \varsigma^{\prime}\left(\gamma_{i}\right)}{\left[\sum_{k} \varsigma\left(\gamma_{k}\right)\right]^{2}}\right\} \\
& =K_{i}(\mathbf{x}) \frac{\varsigma^{\prime}\left(\gamma_{i}\right)}{\sum_{k} \varsigma\left(\gamma_{k}\right)}-\sum_{j} K_{j}(\mathbf{x}) \frac{\varsigma\left(\gamma_{j}\right) \varsigma^{\prime}\left(\gamma_{i}\right)}{\left[\sum_{k} \varsigma\left(\gamma_{k}\right)\right]^{2}} \\
& =K_{i}(\mathbf{x}) \frac{\varsigma^{\prime}\left(\gamma_{i}\right)}{\sum_{k} \varsigma\left(\gamma_{k}\right)}-\left\{\sum_{j} c_{j} K_{j}(\mathbf{x})\right\} \frac{\varsigma^{\prime}\left(\gamma_{i}\right)}{\sum_{k} \varsigma\left(\gamma_{k}\right)} \\
& =\frac{\varsigma^{\prime}\left(\gamma_{i}\right)}{\sum_{k} \varsigma\left(\gamma_{k}\right)}\left\{K_{i}(\mathbf{x})-p(g)\right\}
\end{aligned}
$$

Bearing in mind that the calculations were carried out for a connectionist model of the emission probability $b_{\iota, t}$ associated with the generic $\iota$-th state of the GHMM and evaluated over $t$-th graph in the sequence, and using the symbol $\mathcal{Q}(\iota)$ to denote the subset of the states involved in the current trellis that are instances of the state $\iota$, we can reintroduce the state index and the time index $t$ in the notation, and rewrite Equation (10) as follows:

$$
\frac{\partial b_{q, t}}{\partial \gamma_{i}^{(\iota)}}= \begin{cases}\frac{\varsigma^{\prime}\left(\gamma_{i}^{(\iota)}\right)}{\sum_{k} \varsigma\left(\gamma_{k}^{(\iota)}\right)}\left\{K_{i}^{(\iota)}\left(\mathbf{x}_{t}^{(\iota)}\right)-b_{\iota, t}\right\} & \text { if } q \in \mathcal{Q}(\iota) \\ 0 & \text { otherwise }\end{cases}
$$

where the writings in the form $\gamma_{i}^{(\iota)}, \mathbf{x}_{t}^{(\iota)}$ and $K_{i}^{(\iota)}($.$) (i.e., those having superscript { }^{(\iota)}$ for any value of $\iota=1, \ldots, Q)$ denote the corresponding quantities $\gamma_{i}, \mathbf{x}_{t}$ and $K_{i}($.$) within$ the RBF associated with $\iota$-th state, respectively, according to the previous notation. Substituting Equation (11) into Equation (6) and the latter, in turn, into Equation (4), we obtain the following learning rule for the $i$-th mixing parameter $\gamma_{i}^{(\iota)}$ within the $\iota$-th emission model:

$$
\Delta \gamma_{i}^{(\iota)}=\eta \sum_{q \in \mathcal{Q}(\iota)} \sum_{t} \beta_{q, t} \frac{\alpha_{q, t}}{b_{\iota, t}} \frac{\varsigma^{\prime}\left(\gamma_{i}^{(\iota)}\right)}{\sum_{k} \varsigma\left(\gamma_{k}^{(\iota)}\right)}\left\{K_{i}^{(\iota)}\left(\mathbf{x}_{t}^{(\iota)}\right)-b_{\iota, t}\right\}
$$

where we implicitly exploited the (obvious) fact that $b_{q, t}=b_{\iota, t}$ for all $q \in \mathcal{Q}(\iota)$.

For the means $\mu_{i j}$ and the standard deviations $\sigma_{i j}$ we proceed as follows. Let $\theta_{i j}$ denote the free parameter, i.e. $\mu_{i j}$ or $\sigma_{i j}$, to be estimated. We can write:

$$
\begin{aligned}
\frac{\partial p\left(\phi\left(g \mid \boldsymbol{\theta}_{\phi}\right) \mid \boldsymbol{\theta}_{p}\right)}{\partial \theta_{i j}} & =\frac{\partial \sum_{k=1}^{n} c_{k} K_{k}(\mathbf{x})}{\partial \theta_{i j}} \\
& =c_{i} \frac{\partial K_{i}(\mathbf{x})}{\partial \theta_{i j}}
\end{aligned}
$$


where the calculation of $\frac{\partial K_{i}(\mathbf{x})}{\partial \theta_{i j}}$ can be accomplished as follows. First of all, let us observe that for any real-valued, differentiable function $f($.$) this property holds true:$ $\frac{\partial f(.)}{\partial x}=f(.) \frac{\partial \log [f(.)]}{\partial x}$. As a consequence, from Equation (9] we can write

$$
\frac{\partial K_{i}(\mathbf{x})}{\partial \theta_{i j}}=K_{i}(\mathbf{x}) \frac{\partial}{\partial \theta_{i j}} \sum_{k=1}^{d}\left\{-\frac{1}{2}\left[\log \left(2 \pi \sigma_{i k}^{2}\right)+\left(\frac{x_{k}-\mu_{i k}}{\sigma_{i k}}\right)^{2}\right]\right\} .
$$

For the means, i.e. $\theta_{i j}=\mu_{i j}$, Equation (14) yields

$$
\begin{aligned}
\frac{\partial K_{i}(\mathbf{x})}{\partial \mu_{i j}} & =K_{i}(\mathbf{x}) \frac{\partial}{\partial \mu_{i j}}\left\{-\frac{1}{2}\left(\frac{x_{j}-\mu_{i j}}{\sigma_{i j}}\right)^{2}\right\} \\
& =K_{i}(\mathbf{x}) \frac{x_{j}-\mu_{i j}}{\sigma_{i j}^{2}}
\end{aligned}
$$

which can be substituted into Equation (13), obtaining

$$
\frac{\partial p\left(\phi\left(g \mid \boldsymbol{\theta}_{\phi}\right) \mid \boldsymbol{\theta}_{p}\right)}{\partial \mu_{i j}}=c_{i} K_{i}(\mathbf{x}) \frac{x_{j}-\mu_{i j}}{\sigma_{i j}^{2}} .
$$

Now we can reintroduce the state index $\iota$ and the time index $t$ in the notation, and rewrite the Equation as $\frac{\partial b_{\iota, t}}{\partial \mu_{i j}^{(\iota)}}=c_{i}^{(\iota)} K_{i}^{(\iota)}\left(\mathbf{x}_{t}^{(\iota)}\right) \frac{x_{t j}^{(\iota)}-\mu_{i j}^{(\iota)}}{\sigma_{i j}^{(\iota)}}$, where $x_{t j}^{(\iota)}$ denotes the $j$-th component of the vector $\mathbf{x}_{t}^{(\iota)}$ which represents the $\iota$-th encoding of $t$-th input graph $g_{t}$ within the training sequence, $\sigma_{i j}^{2(\iota)}$ is the $j$-th component of the diagonal of the covariance matrix associated with the $i$-th kernel of $\iota$-th emission PDF, while the other symbols have the same meaning as above. Again, since $\frac{\partial b_{q, t}}{\partial \mu_{i j}^{(\iota)}}=0$ when $q \notin \mathcal{Q}(\iota)$, the expression can be substituted into Equation (6) and the latter, in turn, into Equation (4), obtaining the following learning rule for the $j$-th component of the mean vector $\mu_{i j}^{(\iota)}$ associated with $i$-th kernel function within the $\iota$-th emission model:

$$
\Delta \mu_{i j}^{(\iota)}=\eta \sum_{q \in \mathcal{Q}(\iota)} \sum_{t} \beta_{q, t} \frac{\alpha_{q, t}}{b_{\iota, t}} c_{i}^{(\iota)} K_{i}^{(\iota)}\left(\mathbf{x}_{t}^{(\iota)}\right) \frac{x_{t j}^{(\iota)}-\mu_{i j}^{(\iota)}}{\sigma_{i j}^{2(\iota)}}
$$

where, again, we exploited the fact that $b_{q, t}=b_{\iota, t}$ for all $q \in \mathcal{Q}(\iota)$.

For the covariances, i.e. $\theta_{i j}=\sigma_{i j}$, Equation (14) yields:

$$
\frac{\partial K_{i}(\mathbf{x})}{\partial \sigma_{i j}}=\frac{K_{i}(\mathbf{x})}{\sigma_{i j}}\left\{\left(\frac{x_{j}-\mu_{i j}}{\sigma_{i j}}\right)^{2}-1\right\}
$$

which can be substituted into Equation (13) obtaining

$$
\frac{\partial p\left(\phi\left(g \mid \boldsymbol{\theta}_{\phi}\right) \mid \boldsymbol{\theta}_{p}\right)}{\partial \sigma_{i j}}=c_{i} \frac{K_{i}(\mathbf{x})}{\sigma_{i j}}\left\{\left(\frac{x_{j}-\mu_{i j}}{\sigma_{i j}}\right)^{2}-1\right\}
$$


that, adopting the notation above for expressing the dependence on the generic $\iota$-th state of the GHMM and on the time index $t$, can be substituted into Equation (6) and the latter, in turn, into Equation (4), obtaining the following learning rule:

$$
\Delta \sigma_{i j}^{(\iota)}=\eta \sum_{q \in \mathcal{Q}(\iota)} \sum_{t} \beta_{q, t} \frac{\alpha_{q, t}}{b_{\iota, t}} c_{i}^{(\iota)} \frac{K_{i}^{(\iota)}\left(\mathbf{x}_{t}^{(\iota)}\right)}{\sigma_{i j}^{(\iota)}}\left\{\left(\frac{x_{t j}^{(\iota)}-\mu_{i j}^{(\iota)}}{\sigma_{i j}^{(\iota)}}\right)^{2}-1\right\} .
$$

Finally, let us consider the connection weights $\mathcal{U}=\left\{v_{1}, \ldots, v_{s}\right\}$ within the encoding network. The term $\frac{\partial p\left(\phi\left(g \mid \boldsymbol{\theta}_{\phi}\right) \mid \boldsymbol{\theta}_{p}\right)}{\partial v}$ in Equation (6) can be computed as follows. Applying the chain rule yields:

$$
\frac{\partial p\left(\phi\left(g \mid \boldsymbol{\theta}_{\phi}\right) \mid \boldsymbol{\theta}_{p}\right)}{\partial v}=\frac{\partial p\left(\phi\left(g \mid \boldsymbol{\theta}_{\phi}\right) \mid \boldsymbol{\theta}_{p}\right)}{\partial y} \frac{\partial y}{\partial v}
$$

where $y$ is the output from the unit (in the encoding net) which is fed from connection $v$. The quantity $\frac{\partial y}{\partial v}$ can be easily computed by taking the partial derivative of the activation function associated with the unit itself, as usual. In particular, if $v=v_{\ell m}$ is the connection weight between the generic $m$-th unit in a given layer and $\ell$-th unit in the following layer, s.t. the corresponding outputs are $y_{m}$ and $y_{\ell}$, respectively, we have

$$
\frac{\partial y_{\ell}}{\partial v_{\ell m}}=f_{\ell}^{\prime}\left(a_{\ell}\right) y_{m}
$$

where $y_{\ell}=f_{\ell}\left(a_{\ell}\right)$ and $y_{m}=f_{m}\left(a_{m}\right)$ are the activation functions associated with $\ell$-th unit and $m$-th unit, respectively, and $a_{\ell}$ and $a_{m}$ are the corresponding activations (i.e., inputs), and where $f_{\ell}^{\prime}\left(a_{\ell}\right)$ denotes the derivative of the activation function given.

As regards the quantity $\frac{\partial p\left(\phi\left(g \mid \boldsymbol{\theta}_{\phi}\right) \mid \theta_{\mathbf{p}}\right)}{\partial y}$, we proceed as follows. First of all, let us assume that $v$ feeds the output layer, i.e. it connects a certain hidden unit with $j$-th output unit of the encoding net. In this case, we have $y=x_{j}$, and:

$$
\begin{aligned}
\frac{\partial p\left(\phi\left(g \mid \boldsymbol{\theta}_{\phi}\right) \mid \boldsymbol{\theta}_{p}\right)}{\partial x_{j}} & =\frac{\partial \sum_{i=1}^{n} c_{i} K_{i}(\mathbf{x})}{\partial x_{j}} \\
& =\sum_{i=1}^{n} c_{i} K_{i}(\mathbf{x}) \frac{\partial}{\partial x_{j}} \sum_{k=1}^{d}\left\{-\frac{1}{2}\left[\log \left(2 \pi \sigma_{i k}^{2}\right)+\left(\frac{x_{k} w_{i k}-\mu_{i k}}{\sigma_{i k}}\right)^{2}\right]\right\} \\
& =\sum_{i=1}^{n} c_{i} K_{i}(\mathbf{x})\left\{-\frac{1}{2} \frac{\partial}{\partial x_{j}}\left(\frac{x_{j} w_{i j}-\mu_{i j}}{\sigma_{i j}}\right)^{2}\right\} \\
& =-\sum_{i=1}^{n} c_{i} \frac{K_{i}(\mathbf{x})}{\sigma_{i j}^{2}}\left(x_{j} w_{i j}-\mu_{i j}\right) w_{i j} .
\end{aligned}
$$

Equations (22) and (23) can be substituted into Equation (21) obtaining:

$$
\frac{\partial p\left(\phi\left(g \mid \boldsymbol{\theta}_{\phi}\right) \mid \boldsymbol{\theta}_{p}\right)}{\partial v_{j m}}=-\sum_{i=1}^{n} c_{i} \frac{K_{i}(\mathbf{x})}{\sigma_{i j}^{2}}\left(x_{j} w_{i j}-\mu_{i j}\right) w_{i j} f_{j}^{\prime}\left(a_{j}\right) f_{m}\left(a_{m}\right) .
$$


By defining the quantity:

$$
\delta_{j}=-\sum_{i=1}^{n} c_{i} \frac{K_{i}(\mathbf{x})}{\sigma_{i j}^{2}}\left(x_{j} w_{i j}-\mu_{i j}\right) w_{i j} f_{j}^{\prime}\left(a_{j}\right)
$$

for the generic $j$-th output unit in the encoding network, we can rewrite Equation 24 in the following, compact form:

$$
\frac{\partial p\left(\phi\left(g \mid \boldsymbol{\theta}_{\phi}\right) \mid \boldsymbol{\theta}_{p}\right)}{\partial v_{j m}}=\delta_{j} f_{m}\left(a_{m}\right) .
$$

When $v$ is a hidden weight (say, $v=v_{m \ell}$ where $\ell$ and $m$ are the indexes of generic hidden units connected via $v)$, the quantity $\frac{\partial p\left(\phi\left(g \mid \boldsymbol{\theta}_{\phi}\right) \mid \theta_{\mathbf{p}}\right)}{\partial v_{m \ell}}$ can be obtained applying the usual backpropagation through structures (BPTS) algorithm [8], once the deltas to be backpropagated have been initialized at the output layer via Equation 25). In so doing, a quantity $\delta_{m}$ can be defined for each hidden unit $m$ such that

$$
\frac{\partial p\left(\phi\left(g \mid \boldsymbol{\theta}_{\phi}\right) \mid \boldsymbol{\theta}_{p}\right)}{\partial v_{m \ell}}=\delta_{m} f_{\ell}\left(a_{\ell}\right) .
$$

Substituting Equation (26) or Equation (27), respectively, into Equation (6) yields an overall learning rule for any given weight $v_{i j}^{(\iota)}$ within the encoding network associated with $\iota$-th state of the GHMM in the following, common form:

$$
\Delta v_{i j}^{(\iota)}=\eta \sum_{q \in \mathcal{Q}(\iota)} \sum_{t} \beta_{q, t} \frac{\alpha_{q, t}}{b_{\iota, t}} \delta_{i}^{(\iota)} f_{j}^{(\iota)}\left(a_{j}^{(\iota)}\right)
$$

where the superscript ${ }^{(\iota)}$ has the usual meaning.

\section{Demonstration}

The proposed machine has been implemented, and preliminary experiments were carried out on a graphical sequence recognition task drawn from the Policemen dataset [4]. This dataset features images of synthetically generated policemen, having different color, orientation, position of the arms, etc. Directed ordered acyclic graphs (DOAG) were used for representing the individual images, as explained in [4]. Two-dimensional real-valued labels are associated with the nodes in this DOAG representation. Sequences were generated by taking individual images and creating concatenations of images s.t. a coherent "movement" (as in a cartoon sequence) of the policeman emerged. For instance, the images in a given sequence may represent the policemen gradually rising then lowering his left arm, followed by an analogous movement of the right arm. The task involves 158 sequences overall, belonging to 4 disjoint classes. In turn, each class is further divided into subclasses as follows. Class 1: rotation; subclasses: (1.1) clockwise and (1.2) counter-clockwise. Class 2: shift; subclasses: (2.1) right-left and (2.2) top-down. Class 3: zoom; subclasses: (3.1) zoom-in and (3.2) zoom-out. Class 4: arms movement; subclasses: (4.1) both arms up, (4.2) both arms down, (4.3) right arm up, (4.4) right arm down, (4.5) left arm up, and (4.6) left arm down. Hence, there are 12 classes in total. 

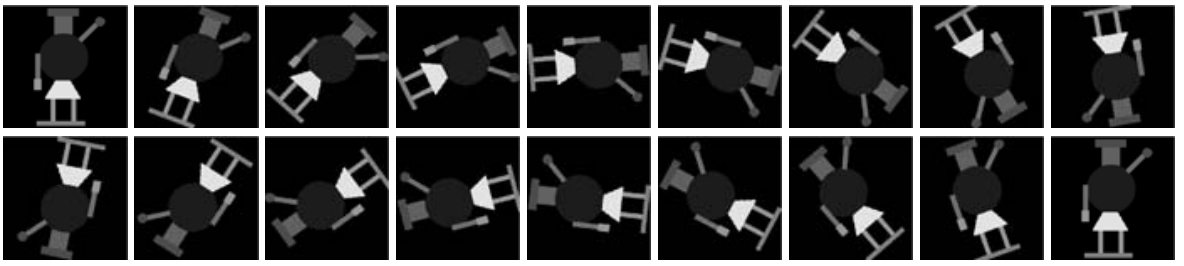

Fig. 1. Sample sequence for subclass 1.1
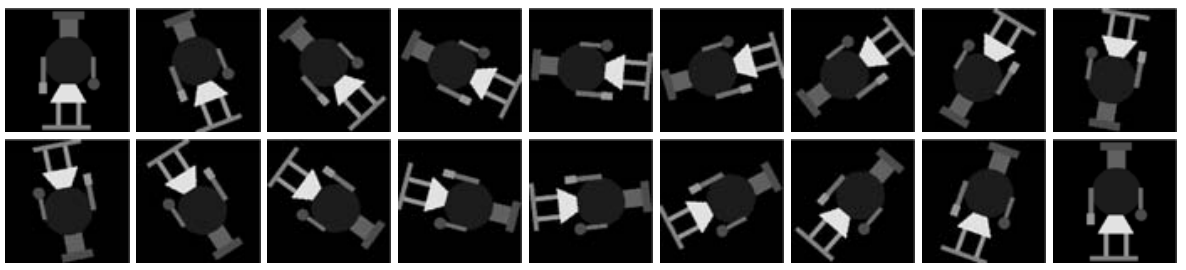

Fig. 2. Sample sequence for subclass 1.2

The length of individual sequences ranged from 10 to 17 graphs. Figures 1 and 2 show two sample sequences from Class 1 . Note that the starting and ending frame of some sequences from different classes were identical, and that for sequences from other classes, some frames from within a sequence were identical. Hence, a good classification can only be obtained if the method can encode the given sequences as a whole.

The dataset was split into a training set (72 sequences, chosen by drawing 6 sequences from each subclass at random) and a test set (all the remaining sequences). Separate left-to-right Markov chains were used for each class, each of them having 4 states. Emission probabilities were modeled with 2 multivariate Gaussian kernels RBFs, and recursive encoding networks having 8 sigmoid hidden neurons, 10 state neurons, encoding dimension of 2, and a maximum out-degree of 6 (please refer to [8] for a description of the meaning of such quantities in recursive nets). System parameters were initialized according to a segmental k-means-like procedure [7]. In the present, illustrative demonstration the proposed training algorithm was applied for 4 iterations (i.e., epochs) using different learning rates (obtained via cross-validation) for the specific parameters involved in the optimization process. Results are reported in Table 1 in terms of recognition accuracy. Although no direct comparison is possible w.r.t. any other benchmark approaches (since, to the best of our knowledge, the present model is the first attempt to dealing with classification of sequences of graphs), it is seen from Table 1 that: (i) the architecture is indeed suitable to graphical sequence modeling; (ii) the training algorithm, whilst focusing on the maximization of the likelihood (of the model given the training sample) criterion, results also in a significant accuracy in terms of the sequence recognition rate criterion; and (iii) comparison between the accuracy on the training and test sets confirms that learning capability of the machine does not prevent emergence of an appreciable generalization capability.

We found that the residual classification error is attributed to two of the subclasses whose properties are such that a correct classification requires the encoding of the associated data labels. Since the labels provide features which are furthest from the output 
Table 1. Recognition accuracy of sequences from the Policemen dataset

\begin{tabular}{lll}
\hline \hline & Accuracy on training set & Accuracy on test set \\
\hline Upon initialization & $51.39 \%$ & $39.53 \%$ \\
After training & $87.50 \%$ & $86.05 \%$ \\
\hline
\end{tabular}

layer, and hence, this implies that the proposed approach priorizes the encoding of structure over the data labels. In general, it can be expected that the classification result will improve further when training is carried out for more iterations, or by adding direct forward links from the labels to the output layer.

\section{Conclusion and On-Going Work}

The paper introduced a first model for learning and classification over sequences of graphs. The architecture relies on an underlying HMM structure, capable of dealing with long-term dependencies in sequential data of arbitrary length. Emission PDFs over GRGs are estimated by means of a combination of recursive encoding nets and constrained RBF-like nets. A global optimization algorithm, aimed at the maximization of the likelihood of the model given the training observation sequences has been developed. Preliminary results confirm that the architecture and the algorithms are effective, both in terms of learning and generalization capabilities. Current efforts are focused on evaluating and further improving the classification and regression ability of the approach, and on its application to more difficult, real-world tasks.

\section{References}

1. Bengio, Y.: Neural Networks for Speech and Sequence Recognition. International Thomson Computer Press, London (1996)

2. Bianchini, M., Maggini, M., Sarti, L.: Object recognition using multiresolution trees. In: Yeung, D.-Y., Kwok, J.T., Fred, A., Roli, F., de Ridder, D. (eds.) SSPR 2006 and SPR 2006. LNCS, vol. 4109, pp. 331-339. Springer, Heidelberg (2006)

3. Di Massa, V., Monfardini, G., Sarti, L., Scarselli, F., Maggini, M., Gori, M.: A comparison between recursive neural networks and graph neural networks. In: World Congress on Computational Intelligence, July 2006, pp. 778-785 (2006)

4. Hagenbuchner, M., Gori, M., Bunke, H., Tsoi, A.C., Irniger, C.: Using attributed plex grammars for the generation of image and graph databases. Pattern Recognition Letters (Special issue on Graph-based Representations) 24(8), 1081-1087 (2002)

5. Haykin, S.: Neural Networks, A Comprehensive Foundation. Macmillan College Publishing Company, Inc., New York (1994)

6. McLachlan, G.J., Basford, K.E. (eds.): Mixture Models: Inference and Applications to Clustering. Marcel Dekker, New York (1988)

7. Rabiner, L.R.: Rabiner. A tutorial on hidden Markov models and selected applications in speech recognition. Proceedings of the IEEE 77(2), 257-286 (1989)

8. Sperduti, A., Starita, A.: Supervised neural networks for the classification of structures. IEEE Transactions on Neural Networks 8(3), 714-735 (1997)

9. Trentin, E., Rigutini, L.: A maximum-likelihood connectionist model for unsupervised learning over graphical domains. In: Alippi, C., Polycarpou, M., Panayiotou, C., Ellinas, G. (eds.) ICANN 2009, Part 1. LNCS, vol. 5768, pp. 40-49. Springer, Heidelberg (2009) 